\title{
Comparison of PICC and TIVAP in chemotherapy for patients with thyroid cancer
}

\author{
FANGMEI QI ${ }^{1}$, HAIRONG CHENG ${ }^{2}$, XIYING YUAN ${ }^{3}$ and LI ZHANG $^{4}$ \\ Departments of ${ }^{1}$ ENT and ${ }^{2}$ Nephrology, Qingdao Municipal Hospital, Qingdao, Shandong 266011; \\ ${ }^{3}$ The Affiliated Central Hospital of Qingdao University, Qingdao, Shandong 266042; \\ ${ }^{4}$ Department of Neurology, East Hospital of Qingdao Municipal Hospital, Qingdao, Shandong 266071, P.R. China
}

Received May 13, 2019; Accepted December 3, 2019

DOI: $10.3892 / \mathrm{ol} .2020 .11732$

\begin{abstract}
Peripherally inserted central catheters (PICC) and totally implantable venous-access ports (TIVAP) were compared in chemotherapy for patients with thyroid cancer. A retrospective analysis was performed on the clinical data of patients with thyroid cancer who were treated with PICC and TIVAP for chemotherapy in Qingdao Municipal Hospital from January 2013 to March 2018. Patients in the PICC and TIVAP groups were compared in terms of the success rate, indwelling time, complications, quality of life and nursing satisfaction. There was no statistically significant difference in the success rate between the two groups $(\mathrm{P}>0.05)$. The indwelling time in the TIVAP group was significantly longer than that in the PICC group $(\mathrm{P}<0.05)$. The incidence rate of complications in the TIVAP group (0\%) was significantly lower than that in the PICC group $(14.58 \%)(\mathrm{P}<0.05)$. The quality of life score in the PICC group was significantly lower than that in the TIVAP group $(\mathrm{P}<0.05)$. There was no statistically significant difference in the nursing satisfaction score between the two groups $(\mathrm{P}>0.05)$. In conclusion, as an ideal venous access to chemotherapy for thyroid cancer, TIVAP has longer indwelling time and fewer adverse reactions and improves the quality of life of the patients.
\end{abstract}

\section{Introduction}

Thyroid cancer is a common malignant endocrine tumor that has a good prognosis, while $15-20 \%$ of patients with differentiated thyroid cancer (DTC) and most patients with anaplastic thyroid cancer (ATC) still have drug resistance to standard regimens, such as radioactive iodine (RAI) treatment (1). The incidence rate of the disease has increased over the past few

Correspondence to: Dr Li Zhang, Department of Neurology, East Hospital of Qingdao Municipal Hospital, 5 Donghai Middle Road, Qingdao, Shandong 266071, P.R. China

E-mail: rre2ey@163.com

Key words: PICC, TIVAP, thyroid cancer, chemotherapy, indwelling time; complication, quality of life decades and nearly tripled in the United States between 1975 and 2009. Despite the increasing incidence rate, the mortality rate of the disease remains stable (2). According to a study, thyroid cancer was the fifth most common cancer threatening women after breast cancer, lung cancer, colon cancer and uterine cancer in Canada in 2016 (3). Thyroid cancer consists of papillary, follicular, medullary and anaplastic thyroid cancers, among which papillary thyroid cancer is the most common one. Tumor is currently treated by chemotherapy, and the patients are treated with liquid medicine during hospitalization for a long time. Permanent needle of deep venipuncture reduces the stimulation to the vein and skin and better injects liquid medicine, which is commonly used in intensive care and conducive to carrying out central venous pressure monitoring, blood transfusion, hemodialysis and total parenteral nutrition (4).

Both peripherally inserted central catheters (PICC) and totally implantable venous-access ports (TIVAP) are currently commonly used techniques for central venous catheterization, while their effects on chemotherapy are different. Studies have shown that PICC based on the patient's condition is usually safe and convenient (5), and may be significantly superior in patients with advanced cancer compared with subcutaneous and peripheral venous catheters. For example, PICC has a strong analgesic effect, causes less surgery-related pain and provides fluid therapy. Additionally, more drugs whose dosages are adjustable are available. It has also been shown that PICC has a high success rate without severe surgery-related complication. According to a study, the success rate of PICC is $\sim 85-95 \%$ in patients without related complications (6). TIVAP, a totally implantable vascular access system, provides long-term venous vascular access and plays a vital role in the treatment of patients with tumors (7). In PICC a thin and long catheter made from a biocompatible material (silicone or polyurethane) is percutaneously inserted into the basal vein or cephalic vein of the forearm or anterior cubital fossa under the guidance of ultrasound or fluoroscopy, and then advanced to the central vein. The tip of the catheter is usually placed at the superior vena cava or the junction between cava and atrium. Compared with other central venous accesses, PICC has fewer complications, such as hemothorax and pneumothorax (8). Therefore, the application values and safety of PICC and TIVAP in chemotherapy 
for patients with thyroid cancer were compared in this study to provide references for clinical practice.

\section{Patients and methods}

General information. A retrospective analysis was performed on the clinical data of 188 patients with thyroid cancer who were treated with PICC or TIVAP for chemotherapy in Qingdao Municipal Hospital (Qingdao, China) from January 2013 to March 2018. The patients were divided into the PICC group and the TIVAP group according to deep venous catheterization. The PICC group consisted of 44 males and 52 females, aged 20-64 years with an average age of 32.35 \pm 8.54 years. According to the pathological type (9), 23 cases were undifferentiated, 33 cases were papillary adenocarcinoma, 24 cases were follicular adenocarcinoma, and 16 cases were medullary carcinoma. The TIVAP group consisted of 40 males and 52 females, aged 21-62 years with an average age of $31.37 \pm 8.91$ years, and 19 cases were undifferentiated, 36 cases were papillary adenocarcinoma, 23 cases were follicular adenocarcinoma, and 14 cases were medullary carcinoma. Inclusion criteria: Patients diagnosed by imaging system and clinicopathology; patients who were aged $>18$ years (5) and had no history of mental illness; patients who were able to communicate normally; patients with complete clinical data; patients who or whose immediate family members signed an informed consent form before the clinical data were collected. Exclusion criteria: Patients with severe cardiac and hepatic dysfunctions; patients who dropped out; patients with malignancies other than thyroid cancer; patients who used to take antiangiogenic drugs; patients with hypertension.

Methods. Patients in the TIVAP group were treated with TIVAP for postoperative intravenous chemotherapy. A Groshong catheter and 3M transparent glue were used. The patient was placed in a horizontal position with the arm extending 190 degrees and the head deviating to the one side in order to determine the puncture site. The biceps circumference, and the distance from the puncture point to the right sternoclavicular joint and from the puncture point down to the third intercostal space were measured and recorded before puncture. After disinfection, draping and local anesthesia were carried out within a radius of $50 \mathrm{~cm}$ of the puncture point, the catheter was inserted into the vein $20.0 \mathrm{~cm}$. After the puncture reached the axillary vein, the lower jaw of the patient approached the shoulder on the side where the catheter was inserted to determine the puncture site, so as to prevent the catheter from entering the internal jugular vein. After puncture, the catheter was fixed with sterile dressings and $3 \mathrm{M}$ glue. After that, chest X-ray was carried out to determine the position of the catheter end. Finally, the patient was instructed to undergo regular review and pay attention to the puncture site for pain and redness.

Patients in the PICC group were treated with PICC for postoperative intravenous chemotherapy. Under the guidance of X-ray fluoroscopy, the catheter was percutaneously inserted into the right subclavian vein. The patient was placed in a horizontal position with the arm extending 190 degrees and the head deviating to the one side in order to determine the puncture site. The biceps circumference, and the distance from the puncture point to the right sternoclavicular joint, and then down to the third intercostal space were measured and recorded before puncture. Disinfection 3 times and local anesthesia were carried out within a radius of $50 \mathrm{~cm}$ of the puncture point. After the right subclavian vein was punctured with a minimally invasive puncture needle, the intravenous indwelling catheter was introduced through the guide wire. After the pillow was removed and the catheter was washed, the catheter was appropriately cut short and the puncture area was bandaged for $24 \mathrm{~h}$. After heparin saline was injected without leakage, the skin incision was sutured and the drainage strip was retained. For both groups of patients, indoor disinfection was carried out before operations that were aseptic during the operation.

Observational indexes. The success rate, indwelling time, complications, quality of life and nursing satisfaction were compared between the two groups. The nursing satisfaction with a total score of 10 points consisted of treatment information, complaint management, physical environment, follow-up, hospitalization and overall service. The higher the score was, the higher the satisfaction was. The quality of life with a total score of 10 points consisted of psychological function, physical function, social function and self-evaluation. The higher the score was, the higher the satisfaction was. The total incidence rate of complications $=$ (cases with local infection + cases with catheter obstruction + cases with catheter leakage + cases with catheter displacement)/total cases.

Statistical methods. SPSS 18.0 (IBM Corp.) was used for statistical analysis, GraphPad Prism 7 for plotting figures. Count data were expressed as number of cases/percentage (n/\%), and Chi-square test was used for comparison of count data between groups. Measurement data were expressed as mean \pm standard deviation (mean $\pm \mathrm{SD}$ ). Student's t-test was used for the comparison of measurement data. $\mathrm{P}<0.05$ indicates a statistically significant difference.

\section{Results}

General information. There was no statistically significant difference between the two groups in terms of sex, age, body weight, clinical staging, pathological types, lymph node metastasis and lesion characteristics $(\mathrm{P}>0.05)$ (Table I).

Comparison of success rate. A total of 95 cases succeeded and 1 case failed in the PICC group, with a success rate of $98.96 \%$. A total of 91 cases succeeded and 1 case failed in the TIVAP group, with a success rate of $98.91 \%$. There was no statistically significant difference between the two groups $\left(\chi^{2}=0.001\right.$, $\mathrm{P}=0.982$ ). More details are shown in Fig. 1.

Comparison of indwelling time. The indwelling time was 98.38 \pm 41.36 days in the TIVAP group, significantly longer than $85.37 \pm 42.48$ days in the PICC group $(\mathrm{P}<0.05)$ (Fig. 2).

Comparison of complications. In the PICC group, there were 3 cases with local infection, 1 with catheter obstruction, 4 with catheter leakage and 6 with catheter displacement, with a total incidence rate of $14.58 \%$. In the TIVAP group, there 
Table I. General information $[\mathrm{n}(\%)]($ mean $\pm \mathrm{SD})$.

\begin{tabular}{|c|c|c|c|c|}
\hline Groups & PICC group $(n=96)$ & TIVAP group $(n=92)$ & $\chi^{2} / \mathrm{t}$ value & P-value \\
\hline Sex & & & 0.105 & 0.745 \\
\hline Male & $44(45.83)$ & $40(43.48)$ & & \\
\hline Female & $52(54.17)$ & $52(54.52)$ & & \\
\hline Age (years) & $32.35 \pm 8.54$ & $31.37 \pm 8.91$ & 0.770 & 0.442 \\
\hline Body weight (kg) & $61.98 \pm 5.24$ & $63.45 \pm 6.78$ & 0.097 & 0.097 \\
\hline Clinical staging & & & 1.355 & 0.244 \\
\hline Stages I-II & $43(44.79)$ & $38(41.30)$ & & \\
\hline Stages III-IV & $53(55.21)$ & $54(58.70)$ & & \\
\hline Pathological types & & & 1.241 & 0.743 \\
\hline Undifferentiated & $23(23.95)$ & $19(20.65)$ & & \\
\hline Polypoid adenocarcinoma & $33(34.38)$ & $38(41.30)$ & & \\
\hline Follicular carcinoma & $24(25.00)$ & $23(25.00)$ & & \\
\hline Medullary carcinoma & $16(16.67)$ & $12(13.05)$ & & \\
\hline Lymph node metastasis & & & 0.637 & 0.425 \\
\hline Yes & $29(30.21)$ & $23(25.00)$ & & \\
\hline No & $67(69.79)$ & $69(75.00)$ & & \\
\hline Lesion characteristic & & & 0.528 & 0.468 \\
\hline Uninodular & $34(35.42)$ & $28(30.43)$ & & \\
\hline Multinodular & $62(64.58)$ & $64(69.57)$ & & \\
\hline
\end{tabular}

PICC, peripherally inserted central catheters; TIVAP, totally implantable venous-access ports.

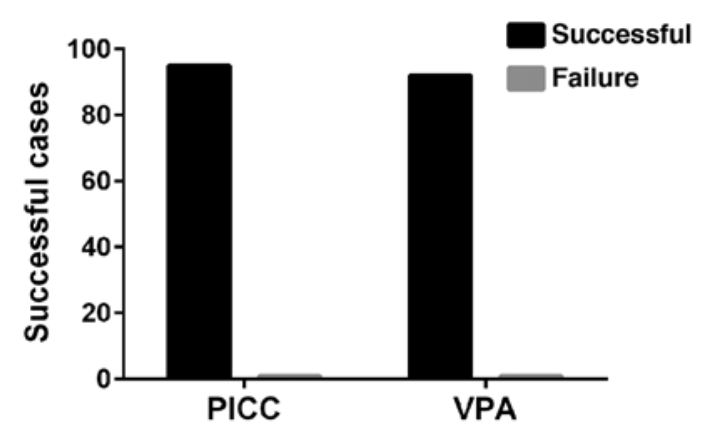

Figure 1. Comparison of success rate. There was no statistically significant difference in the success rate between the two groups $\left(\chi^{2}=0.001, \mathrm{P}=0.982\right)$.

was no complication mentioned above, with a total incidence rate of $0 \%$. The incidence rate of complications in the TIVAP group $(0 \%)$ was significantly lower than that in the PICC group $(14.58 \%)(\mathrm{P}<0.05)$ (Table II).

Comparison of quality of life score. In the PICC group, the physical function was $8.21 \pm 0.66$ points, the psychological function was $8.17 \pm 0.55$ points, the social function was $8.65 \pm 0.63$ points and the self-evaluation was $8.42 \pm 0.54$ points In the TIVAP group, those were respectively $9.17 \pm 0.61$, $9.22 \pm 0.58,9.59 \pm 0.58$ and $9.38 \pm 0.61$ points. The quality of life scores in the PICC group were significantly lower than those in the TIVAP group $(\mathrm{P}<0.05)$ (Fig. 3).

Comparison of nursing satisfaction score. In the PICC group, the treatment information was $9.36 \pm 0.76$ points, the complaint

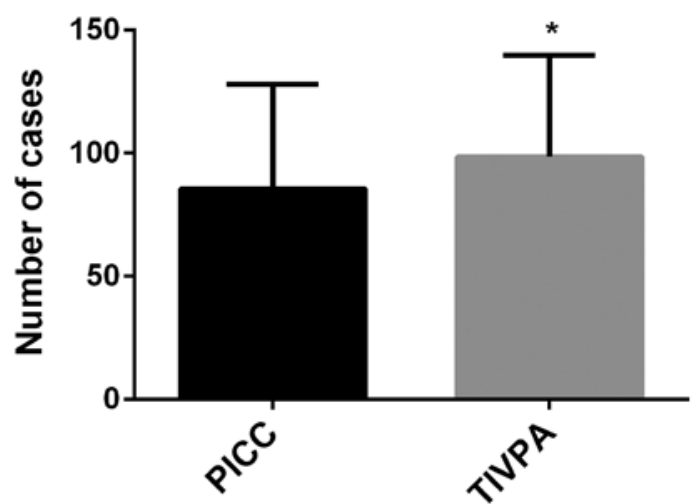

Figure 2. Comparison of indwelling time. The indwelling time in the TIVAP group was significantly longer than that in the PICC group $(\mathrm{P}<0.05)$. ${ }^{*} \mathrm{P}<0.05$ compared with the PICC group. PICC, peripherally inserted central catheters. TIVAP, totally implantable venous-access ports.

management was $9.67 \pm 0.73$ points, the follow-up was $9.42 \pm 0.86$ points, the hospitalization was $9.62 \pm 0.65$ points and the overall service was $9.73 \pm 0.86$ points. In the TIVAP group, those were, respectively, 9.65 $\pm 0.77,9.73 \pm 0.86,9.73 \pm 0.86$, $9.52 \pm 0.83$ and $9.26 \pm 0.75$ points. There was no statistically significant difference in the nursing satisfaction scores between the two groups ( $\mathrm{P}>0.05)$ (Fig. 4).

\section{Discussion}

As a common malignant endocrine tumor that has an increasing incidence rate, a high recurrence rate or high persistence but 
Table II. Comparison of complications [n(\%)].

\begin{tabular}{lccccc}
\hline Groups & $\begin{array}{c}\text { Local } \\
\text { infection }\end{array}$ & $\begin{array}{c}\text { Catheter } \\
\text { obstruction }\end{array}$ & $\begin{array}{c}\text { Catheter } \\
\text { leakage }\end{array}$ & $\begin{array}{c}\text { Catheter } \\
\text { displacement }\end{array}$ & $\begin{array}{c}\text { Total } \\
\text { incidence rate }\end{array}$ \\
\hline PICC group (n=96) & $3(3.13)$ & $1(1.04)$ & $4(4.16)$ & $6(6.25)$ & $14(14.58 \%)$ \\
TIVAP group (n=92) & 0 & 0 & 0 & 0 & 0 \\
$\chi^{2}$ value & - & - & - & - & 14.50 \\
P-value & - & - & - & - & 0.001 \\
\hline
\end{tabular}

PICC, peripherally inserted central catheters; TIVAP, totally implantable venous-access ports.

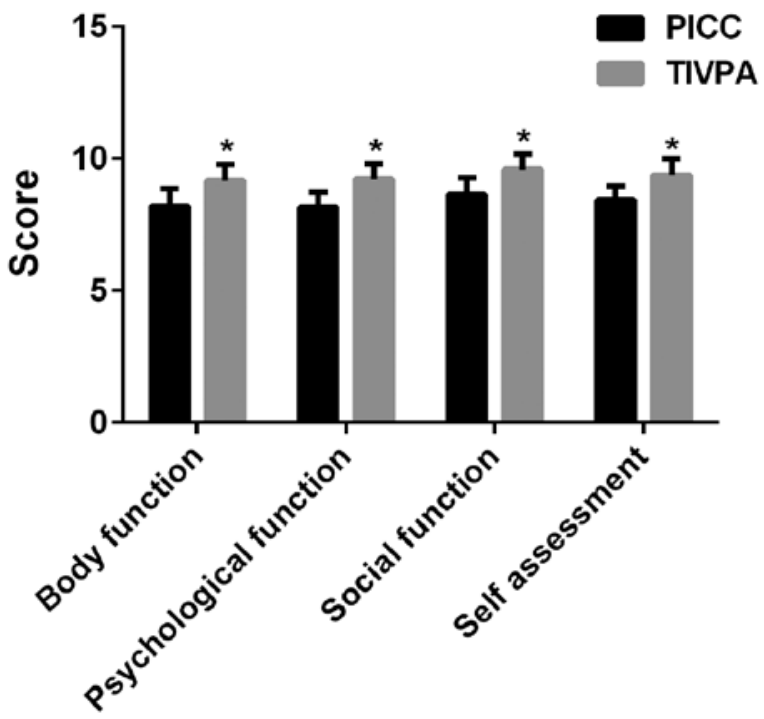

Figure 3. Comparison of quality of life score. The quality of life scores in the PICC group were significantly lower than those in the TIVAP group $(\mathrm{P}<0.05)$. ${ }^{*} \mathrm{P}<0.05$ compared with the PICC group. PICC, peripherally inserted central catheters; TIVAP, totally implantable venous-access ports.

a low mortality rate, thyroid cancer has different histological types and subtypes, as well as cell sources, characteristics and prognoses (10). Central venous access devices (CVADs) provide an access to the larger vascular system for peripheral treatment, contraindication treatment, long-term treatment, venous monitoring and blood sampling. Patients who require CVADs are heterogeneous, and they have different ages and acute and chronic diseases, as well as hospital and community care. CVADs such as long- or short-term treatment and continuous or intermittent treatment are alternative and patients can choose types based on their conditions (11-14). While CVADs are clinically popular, their complications such as blood infection are still common and considered to cause damage to patients, negatively affecting the healthcare cost of the patients. At present, the prevention of CVADs-related injuries is taken into consideration of healthcare researchers, clinicians and patients (15). PICC is commonly used medical equipment for saving life (16). Usually inserted into peripheral deep veins (bonethirst, brachium, head and armpit), the catheters are used for blood sampling, drug, liquid and nutrient delivery through the superior vena cava (17). PICC is suitable for long-term or frequent treatment, chemotherapy and infusion of irritant drugs, especially for patients who cannot be treated with PICC

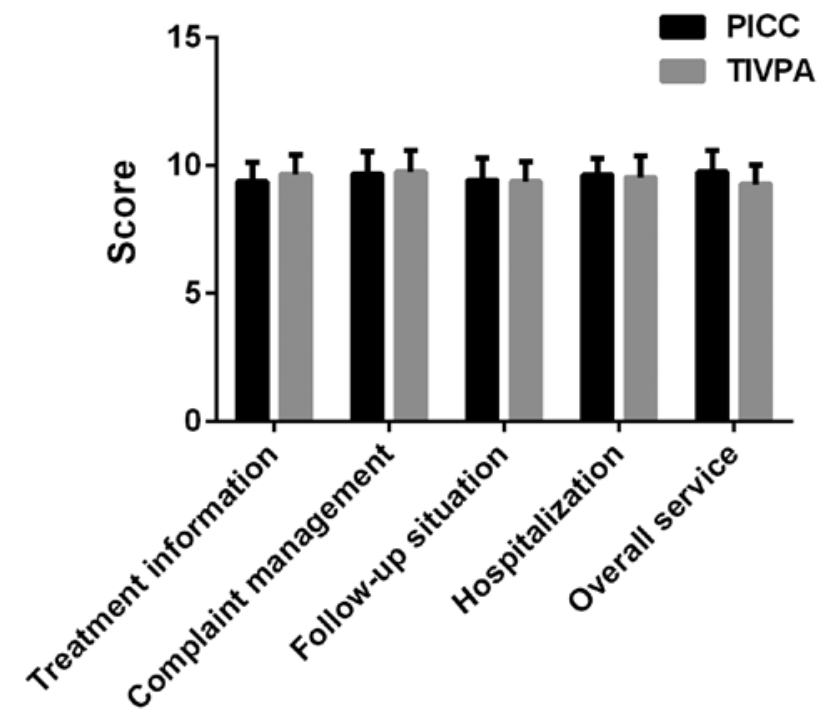

Figure 4. Comparison of nursing satisfaction score. There was no statistically significant difference in the nursing satisfaction scores between the two groups $(\mathrm{P}>0.05)$.

through the vein (18). However, the failure of PICC leads to poor experience, including stabbing pain and increase in hospital stay, equipment cost and work (19). TIVAP is a widely used intermittent central venous access, especially suitable for patients with cancers, and its ports have higher safety margin compared with other long-term venous access devices (20). It is generally used for patients who require long-term or repeated treatment, such as antitumor chemotherapy, parenteral nutrition and blood transfusion. TIVAP causes infection and even endangers lives even though the risk of infection is low. Pocket or subcutaneous tunnel infection is probably caused by TIVAP that should be removed immediately $(21)$. PICC $(22,23)$ and TIVAP $(24,25)$ provide effective deep venous accesses for patients with tumors, but they have their own advantages and disadvantages. Therefore, the application values and safety of PICC and TIVAP in chemotherapy for patients with thyroid cancer were compared in this study to provide references for clinical practice.

In this study, the success rate in the PICC group was $98.96 \%(95 / 96)$ and that in the TIVAP group was $98.91 \%$ (91/92), without statistically significant difference; the indwelling time in the TIVPA group was significantly longer than that in the PICC group; the incidence rate of complications in the TIVPA group was significantly lower than that 
in the PICC group. A retrospective study of 1,254 patients with TIVAP implantation showed that the success rate was $99.9 \%$ and the incidence of complications was 5\% (26). Another study observed 1,183 patients implanted with TIVAP for 30 days and found that only $0.6 \%$ of patients had catheter-related infections (27). A recent study showed that PICC had a higher risk of catheter-related deep vein thrombosis and other adverse events than TIVAP (28). Our results are similar to the above findings, which further suggests that TIVAP is more secure and effective than PICC. In this study, the physical function, psychological function, social function and self-evaluation scores in the TIVAP group were significantly higher than those in the PICC group, whereas there was no statistically significant difference in the nursing satisfaction score between the two groups. Biacchi et al (29) found that compared with PICC, TIVAP management was simpler, provided better quality of life for patients and reduced complications. In a study by Patel et al (30), TIVAP was superior to PICC in the quality of life but inferior in medical expenses. PICC has a high incidence rate of infection due to the indwelling catheter in vitro, so the patients should go to the hospital regularly for local skin management in order to prevent infection.

In conclusion, both PICC and TIVAP provide a deep venous access, but TIVAP has a low incidence rate of complications, higher safety and better improvement in quality of life. Therefore, TIVAP is better for chemotherapy in patients with thyroid cancer, worthy of clinical promotion.

\section{Acknowledgements}

Not applicable.

\section{Funding}

No funding was received.

\section{Availability of data and materials}

The datasets used and/or analyzed during the present study are available from the corresponding author on reasonable request.

\section{Authors' contributions}

FQ compared the complications and the quality of life and wrote the manuscript. HC analyzed and interpreted the patient data. XY performed the experiment and designed the study. $\mathrm{LZ}$ was responsible for the analysis and discussion of the data. All authors read and approved the final manuscript.

\section{Ethics approval and consent to participate}

The study was approved by the Ethics Committee of Qingdao Municipal Hospital (Qingdao, China). Patients who participated in this research, signed an informed consent and had complete clinical data.

\section{Patients consent for publication}

Not applicable.

\section{Competing interests}

The authors declare that they have no competing interests.

\section{References}

1. Asna N, Livoff A, Batash R, Debbi R, Schaffer P, Rivkind T and Schaffer M: Radiation therapy and immunotherapy-a potential combination in cancer treatment. Curr Oncol 25: e454-e460, 2018.

2. Pajamäki N, Metso S, Hakala T, Ebeling T, Huhtala H, Ryödi E, Sand J, Jukkola-Vuorinen A, Kellokumpu-Lehtinen PL and Jaatinen P: Long-term cardiovascular morbidity and mortality in patients treated for differentiated thyroid cancer. Clin Endocrinol (Oxf) 88: 303-310, 2018.

3. Topstad D and Dickinson JA: Thyroid cancer incidence in Canada: A national cancer registry analysis. CMAJ Open 5: E612-E616, 2017.

4. Hazama S, Tamada K, Yamaguchi Y, Kawakami Y and Nagano H: Current status of immunotherapy against gastrointestinal cancers and its biomarkers: Perspective for precision immunotherapy. Ann Gastroenterol Surg 2: 289-303, 2018.

5. Gao Y, Liu Y, Ma X, Wei L, Chen W and Song L: The incidence and risk factors of peripherally inserted central catheter-related infection among cancer patients. Ther Clin Risk Manag 11: 863-871, 2015

6. Osmani L, Askin F, Gabrielson E and Li QK: Current WHO guidelines and the critical role of immunohistochemical markers in the subclassification of non-small cell lung carcinoma (NSCLC): Moving from targeted therapy to immunotherapy. Semin Cancer Biol 52: 103-109, 2018.

7. Cheng F, Liang H, Butte AJ, Eng C and Nussinov R: Personal mutanomes meet modern oncology drug discovery and precision health. Pharmacol Rev 71: 1-19, 2019.

8. Johansson E, Hammarskjöld F, Lundberg D and Arnlind MH: Advantages and disadvantages of peripherally inserted central venous catheters (PICC) compared to other central venous lines: A systematic review of the literature. Acta Oncol 52: 886-892, 2013.

9. Cooper DS, Doherty GM, Haugen BR, Kloos RT, Lee SL, Mandel SJ, Mazzaferri EL, McIver B, Pacini F, Schlumberger M, et al; American Thyroid Association (ATA) Guidelines Taskforce on Thyroid Nodules and Differentiated Thyroid Cancer: Revised American Thyroid Association management guidelines for patients with thyroid nodules and differentiated thyroid cancer: The American Thyroid Association (ATA) guidelines taskforce on thyroid nodules and differentiated thyroid cancer. Thyroid 19: 1167-1214, 2009.

10. Xing M: Molecular pathogenesis and mechanisms of thyroid cancer. Nat Rev Cancer 13: 184-199, 2013.

11. Loveday HP, Wilson JA, Pratt RJ, Golsorkhi M, Tingle A, Bak A, Browne J, Prieto J and Wilcox M; UK Department of Health: epic3: National evidence-based guidelines for preventing healthcare-associated infections in NHS hospitals in England. J Hosp Infect 86 (Suppl 1): S1-S70, 2014.

12. Taylor JE, McDonald SJ and Tan K: Prevention of central venous catheter-related infection in the neonatal unit: A literature review. J Matern Fetal Neonatal Med 28: 1224-1230, 2015.

13. Davis MH: Pediatric central venous catheter management: A review of current practice. JAVA 18: 93-98, 2013.

14. McMullan C, Propper G, Schuhmacher C, Sokoloff L, Harris D, Murphy P and Greene WH: A multidisciplinary approach to reduce central line-associated bloodstream infections. Jt Comm J Qual Patient Saf 39: 61-69, 2013.

15. Broadhurst D, Moureau N and Ullman AJ; World Congress of Vascular Access (WoCoVA) Skin Impairment Management Advisory Panel: Management of central venous access device-associated skin impairment: An evidence-based algorithm. J Wound Ostomy Continence Nurs 44: 211-220, 2017.

16. Schiffer CA, Mangu PB, Wade JC, Camp-Sorrell D, Cope DG, El-Rayes BF, Gorman M, Ligibel J, Mansfield P and Levine M: Central venous catheter care for the patient with cancer: American Society of Clinical Oncology clinical practice guideline. J Clin Oncol 31: 1357-1370, 2013.

17. Cotogni $\mathrm{P}$ and Pittiruti M: Focus on peripherally inserted central catheters in critically ill patients. World J Crit Care Med 3: 80-94, 2014. 
18. Chopra V, Flanders SA, Saint S, Woller SC, O'Grady NP Safdar N, Trerotola SO, Saran R, Moureau N, Wiseman S, et al; Michigan Appropriateness Guide for Intravenouse Catheters (MAGIC) Panel: The Michigan Appropriateness Guide for Intravenous Catheters (MAGIC): Results from a multispecialty panel using the RAND/UCLA appropriateness method. Ann Intern Med 163 (Suppl 6): S1-S40, 2015.

19. Walz JM, Memtsoudis SG and Heard SO: Prevention of central venous catheter bloodstream infections. J Intensive Care Med 25: 131-138, 2010.

20. Pinelli F, Cecero E, Degl'Innocenti D, Selmi V, Giua R, Villa G, Chelazzi C, Romagnoli S and Pittiruti M: Infection of totally implantable venous access devices: A review of the literature. J Vasc Access 19: 230-242, 2018.

21. Shang M, Chang C, Pei Y, Guan Y, Chang J and Li H: Potential management of circulating tumor DNA as a biomarker in triple-negative breast cancer. J Cancer 9: 4627-4634, 2018.

22. Hatakeyama N, Hori T, Yamamoto M, Mizue N, Inazawa N, Igarashi K, Tsutsumi H and Suzuki N: An evaluation of peripherally inserted central venous catheters for children with cancer requiring long-term venous access. Int J Hematol 94: 372-377, 2011.

23. Macmillan T, Pennington M, Summers JA, Goddard K, Zala D, Herz N, Peacock JL, Keevil S and Chalkidou A: SecurAcath for securing peripherally inserted central catheters: A NICE medical technology guidance. Appl Health Econ Health Policy 16: 779-791, 2018.

24. Ignatov A, Hoffman O, Smith B, Fahlke J, Peters B, Bischoff J and Costa SD: An 11-year retrospective study of totally implanted central venous access ports: Complications and patient satisfaction. Eur J Surg Oncol 35: 241-246, 2009.

25. Subramaniam A, Kim KH, Bryant SA, Kimball KJ, Huh WK, Straughn JM, Estes JM and Alvarez RD: Incidence of mechanical malfunction in low-profile subcutaneous implantable venous access devices in patients receiving chemotherapy for gynecologic malignancies. Gynecol Oncol 123: 54-57, 2011.
26. Ahn SJ, Kim HC, Chung JW, An SB, Yin YH, Jae HJ and Park JH: Ultrasound and fluoroscopy-guided placement of central venous ports via internal jugular vein: Retrospective analysis of 1254 port implantations at a single center. Korean J Radiol 13: 314-323, 2012.

27. Covey AM, Toro-Pape FW, Thornton RH, Son C, Erinjeri J, Sofocleous CT, Brody LA, Brown KT, Sepkowitz KA and Getrajdman GI: Totally implantable venous access device placement by interventional radiologists: Are prophylactic antibiotics necessary? J Vasc Interv Radiol 23: 358-362, 2012.

28. Taxbro K, Hammarskjöld F, Thelin B, Lewin F, Hagman H, Hanberger $\mathrm{H}$ and Berg S: Clinical impact of peripherally inserted central catheters vs implanted port catheters in patients with cancer: An open-label, randomised, two-centre trial. Br J Anaesth 122: 734-741, 2019.

29. Biacchi D, Sammartino P, Sibio S, Accarpio F, Cardi M, Sapienza P, De Cesare A, Atta JM, Impagnatiello A and Di Giorgio A: Does the implantation technique for totally implantable venous access ports (TIVAPs) influence long-term outcome? World J Surg 40: 284-290, 2016.

30. Patel GS, Jain K, Kumar R, Strickland AH, Pellegrini L, Slavotinek J, Eaton M, McLeay W, Price T, Ly M, et al: Comparison of peripherally inserted central venous catheters (PICC) versus subcutaneously implanted port-chamber catheters by complication and cost for patients receiving chemotherapy for non-haematological malignancies. Support Care Cancer 22: 121-128, 2014.

This work is licensed under a Creative Commons Attribution-NonCommercial-NoDerivatives 4.0 International (CC BY-NC-ND 4.0) License. 